\title{
Relevância e lugar dos sistemas alimentares na sindemia da COVID-19
}

\author{
Relevance and place of food systems in the \\ COVID-19 syndemic
}

\author{
Relevancia y lugar de los sistemas alimentarios \\ en la sindemia de COVID-19
}

José Patrício Bispo Júnior 1
Djanilson Barbosa dos Santos 2

doi: 10.1590/0102-311X00266721
Diante da complexidade da sindemia da COVID-19 e da natureza desconhecida dos efeitos futuros, é salutar o aprofundamento do debate sobre o tema, assim como o desenvolvimento de outros estudos teóricos e empíricos que possam evidenciar a magnitude e potenciais soluções para o problema. A partir do modelo proposto por nós 1, um grupo de autores escreveu uma Carta às Editoras com reflexões sobre o espaço dos sistemas alimentares atuais na sindemia da COVID-192.

Inicialmente, cabe um esclarecimento sobre o seguinte trecho da carta "os autores analisaram a interdependência existente entre doenças infecciosas e parasitárias (DIP), doenças crônicas não transmissíveis (DCNT) e saúde mental, sendo a COVID-19 um resultado dessa interação" 2 (p. 1). Conforme exposto, afirma-se que a COVID-19 é resultante da interação entre os referidos grupos de doenças, o que não corresponde ao apresentado por nós. Naturalmente, a COVID-19 não é o resultado da interação entre outras doenças. Trata-se de um agravo com etiologia e fisiopatologia bastante específicas e com desenvolvimento que independe da ocorrência de outras doenças.

O modelo proposto por nós considera que a interação sinérgica entre DIP, DCNT, problemas de saúde mental e COVID-19 potencializa a incidência e desencadeia repercussões mais graves tanto da COVID-19 como das demais doenças 1 . Assim, apresentamos a complexidade e a grande capacidade de impacto da sindemia da COVID-19 para além dos aspectos biológicos. Chamamos a atenção para a necessidade de abordagem ampliada do problema com intervenções que devem ir muito além das ações tradicionais de saúde pública focadas em mecanismos de mitigação da transmissibilidade do vírus 1. Isto porque não se pode desconsiderar a determinação social da doença causada pelo SARS-CoV-2, que segue impactando com maior força a vida dos mais necessitados 3 .

Esclarecida essa questão semântica, passaremos ao diálogo sobre a sugestão apresentada na carta, na qual os autores propõem que os sistemas alimentares insustentáveis devam ser integrados ao modelo teórico da COVID-19 como sindemia. Tal reflexão é relevante e constitui-se numa oportunidade para o aprofundamento da compreensão do modelo.

Consideramos muito pertinente os autores trazerem para o debate da COVID-19 os sistemas alimentares atuais. No contexto global, observa-se um quadro de concomitância entre a elevada prevalência de obesidade e da persistência e incremento da desnutrição ${ }^{4}$. Este cenário foi denominado dupla carga global da desnutrição, em que as deficiências nutricionais e o aumento do excesso de peso ocorre principalmente em decorrência das rápidas mudanças nos sistemas alimentares 5 . No Brasil, a
1 Instituto Multidisciplinar de Saúde, Universidade Federal da Bahia, Vitória da Conquista, Brasil.

2 Centro de Ciências da Saúde, Universidade Federal do Recôncavo da Bahia, Santo Antônio de Jesus, Brasil.

Correspondência J. P. Bispo Júnior Rua Hormindo Barros 58, Vitória da Conquista, BA 45029-094, Brasil. jpatricio@ufba.br 
fome e a insegurança alimentar são questões persistentes e estruturais resultantes da intensa desigualdade de renda que perdura no país 6 .

Destarte, a segurança alimentar se constitui como importante determinante da saúde. Himmelgreen et al. 7 ressaltam as quatro dimensões que possibilitam identificar o nível de segurança alimentar de indivíduos, famílias e comunidades: disponibilidade, acesso, utilização e estabilidade. No âmbito dos sistemas alimentares, o não atendimento a essas questões contribui para o quadro de desnutrição e de insegurança alimentar. Este cenário propicia o elevado consumo de bebidas e alimentos ultraprocessados de baixo custo, ricos em gorduras e carboidratos refinados, e favorece o desenvolvimento da obesidade e da carência nutricional 5 .

É também destacado que os sistemas alimentares atuais participam significativamente do esgotamento dos recursos naturais e das mudanças climáticas, o que reforça a característica de insustentabilidade 2. Deste modo, são muito bem-vindas as contribuições que fazem refletir como as atuais configurações dos sistemas alimentares influenciam a saúde das populações e a degradação do meio ambiente.

$\mathrm{Na}$ carta 2, não está exatamente claro se a sugestão é para a inclusão dos sistemas alimentares como um grupo de causas, assim como as DIP, as DCNT e os problemas de saúde mental. Caso seja, consideramos que tal proposição mostra-se deslocada e não apropriada para o modelo teórico por nós defendido. Isso não significa desconsiderar a relevância dos sistemas alimentares e da segurança alimentar. Na verdade, ressaltamos a importância de compreender a abrangência e o espaço que os aspectos alimentares e nutricionais, incluindo o sistema produtivo, ocupam no modelo.

No artigo, nós não abordamos especificamente a discussão sobre os sistemas alimentares, o que talvez tenha motivado o questionamento da necessidade de sua integração ao modelo. No entanto, os aspectos referentes à alimentação e nutrição são abordados no texto e constituem-se como elementos integrantes do modelo sindêmico.

Como ressaltado na carta 2, os sistemas alimentares estão associados com os três grupos principais de doenças: DIP, DCNT e problemas de saúde mental. Também a Comissão Global da The Lancet sobre obesidade adverte que a nutrição inadequada em todas as suas formas, incluindo obesidade, é a principal causa dos problemas de saúde em todo o mundo ${ }^{8}$. Assim, é oportuno situar que os problemas de alimentação e nutrição são macrodeterminantes estruturais que favorecem a ocorrência de doenças dos diversos grupos. Pari passu, também se constituem como contexto social adverso que potencializa a interação sinérgica e o agravamento de todas as doenças envolvidas na sindemia da COVID-19.

Um importante aspecto da teoria é que os processos sindêmicos não ocorrem apenas no nível biológico, relacionados à interação entre diferentes patologias. No nosso artigo, a relevância e a predominância dos aspectos sociais como elementos condicionantes da sindemia são devidamente destacadas 1 . Assim, fatores como a pobreza e os aspectos nutricionais podem constituir-se como muito mais decisivos para o desenvolvimento e potencialização dos efeitos sindêmicos do que os aspectos biológicos 9 .

Neste sentido, esclarecemos que os sistemas alimentares e a segurança alimentar e nutricional já estão integrados no modelo teórico, uma vez que fazem parte do contexto social que potencializa e no qual se desenvolve a sindemia. Na Figura 2 de nosso ensaio 1, nós apresentamos uma representação esquemática do modelo teórico da sindemia. Nesta, é possível visualizar que a interação sinérgica entre os grupos de doença se desenvolve a partir do contexto social e econômico. É precisamente neste contexto que se situam os sistemas alimentares atuais que favorecem, junto a outros determinantes, a sindemia da COVID-19. 


\section{Colaboradores}

J. P. Bispo Júnior participou da redação do manuscrito e revisão crítica. D. B. Santos participou da revisão crítica do manuscrito. Ambos os autores aprovaram a versão final.
1. Bispo Júnior JP, Santos DB. COVID-19 como sindemia: modelo teórico e fundamentos para a abordagem abrangente em saúde. Cad Saúde Pública 2021; 37:e0119021.

2. Machado AD, Marchioni DML, Carvalho AM. A insustentabilidade dos sistemas alimentares atuais deve ser integrada no entendimento da COVID-19 como uma sindemia. Cad Saúde Pública 2021; 37:00253221.

3. Bispo Júnior JP, Morais MB. Participação comunitária no enfrentamento da COVID-19: entre o utilitarismo e a justiça social. Cad Saúde Pública 2020; 36:e00151620.

4. Wells JCK, Marphatia AA, Amable G, Siervo M, Friis H, Miranda JJ, et al. The future of human malnutrition: rebalancing agency for better nutritional health. Global Health 2021; 17:119.

5. Popkin BM, Corvalan C, Grummer-Strawn LM. Dynamics of the double burden of malnutrition and the changing nutrition reality. Lancet 2020; 395:65-74.

\section{Informações adicionais}

ORCID: José Patrício Bispo Júnior (0000-00034155-9612); Djanilson Barbosa dos Santos (00000002-6128-1155).

6. Brito FRSS, Baptista TWF. Sentidos e usos da fome no debate político brasileiro: recorrência e atualidade. Cad Saúde Pública 2021; 37:e00308220.

7. Himmelgreen D, Romero-Daza N, Heuer J, Lucas W, Salinas-Miranda AA, Stoddard T. Using syndemic theory to understand food insecurity and diet-related chronic diseases. Soc Sci Med 2020; [Epub ahead of print].

8. Swinburn BA, Kraak VI, Allender S, Atkins VJ, Baker PI, Bogard JR, et al. The global syndemic of obesity, undernutrition, and climate change: The Lancet Commission report. Lancet 2019; 393:791-846.

9. Singer M, Bulled N, Ostrach B, Mendenhall E. Syndemics and the biosocial conception of health. Lancet 2017; 389:941-50. 